\title{
Modeling the Defect Inspection Sensitivity of a Confocal Microscope
}

\author{
E. M. Gullikson ${ }^{a^{*}}$, E. Tejnil ${ }^{\mathrm{b}}$, K.-Y. Tsai ${ }^{\mathrm{b}}$, A. R. Stivers ${ }^{\mathrm{b}}$, H. Kusunose ${ }^{\mathrm{c}}$ \\ ${ }^{a}$ Center for X-Ray Optics, Lawrence Berkeley National Laboratory, Berkeley, CA 94720 \\ ${ }^{\mathrm{b}}$ Intel Corporation, SC1-03, 2200 Mission College Ave., Santa Clara, CA 95054 \\ ${ }^{c}$ Lasertec Corporation, Yokohama, 223-8551 Japan
}

\begin{abstract}
The ability of a confocal microscope to inspect for defects on EUVL mask blanks has been investigated both experimentally and theoretically. A model was developed to predict the image contrast of a confocal microscope. Measurements were made on PSL spheres and programmed multilayer defects using a Lasertec M1350 operating with $488 \mathrm{~nm}$ light. The images obtained of PSL spheres on both fused silica and multilayer-coated blanks are accurately predicted with the model using no adjustable parameters. Good agreement is also demonstrated for the modeling of multilayer defects. Predictions are made for the expected increase in contrast at the shorter wavelength of $266 \mathrm{~nm}$. Substrate roughness contributes to the "noise" which limits the sensitivity to small defects. The contrast fluctuations due to roughness have been modeled using a simple single surface approximation. The model has been validated with measurements on substrates with varying degrees of roughness. The contribution of mask roughness to the sensitivity of a $266 \mathrm{~nm}$ tool is estimated.
\end{abstract}

Keywords: EUVL, mask blank, defect, inspection, sensitivity, confocal microscope

\section{INTRODUCTION}

Producing a defect free mask is one of the key challenges for the successful development of extreme ultraviolet lithography, EUVL. The ability to inspect for defects both on the mask substrate (before multilayer coating) and on the mask blank is essential. Actinic defect inspection operating at $13.5 \mathrm{~nm}$ is being investigated ${ }^{1-2}$. However, the inspection task would be simplified if a conventional tool operating at a longer wavelength could be used. Confocal scanning optical microscopy is a promising inspection technique ${ }^{3}$ and commercially available tools have been installed at EUV mask pilot lines in Santa Clara, California ${ }^{4}$ and Albany, New York ${ }^{5}$.

EUV lithography uses a reflective mask with a Mo/Si multilayer coating to provide high reflectivity at the operating wavelength of $13.5 \mathrm{~nm}$. Defects occurring below or within the multilayer coating can lead to printable defects with a height of around $1 \mathrm{~nm}$ at the surface of the mask ${ }^{6}$. An inspection tool must be capable of detecting these defects with a high capture rate in a reasonable amount of time. The goal of this work was to develop a model for the sensitivity of a confocal microscope and use it to assess the ability of this technique to inspect for these critical defects.

Polystyrene Latex (PSL) spheres are readily available with known sizes and are often used to calibrate the sensitivity of defect inspection tools. For this reason, a model was developed for the imaging of a sphere on a surface and compared to measurements of PSL spheres on both fused silica substrates and multilayer-coated mask blanks. Multilayer defects were modeled using a simple single surface approximation and compared with measurements on a programmed defect mask. The M1350 has an approximate sensitivity of $50 \mathrm{~nm}$ PSL on a fused silica substrate, and $80 \mathrm{~nm}$ PSL on a Mo/Si coated mask blank. It is capable of detecting Gaussian defects with a full-width at half maximum (FWHM) of $60 \mathrm{~nm}$ and height of $10 \mathrm{~nm}$ with greater than $90 \%$ capture rate ${ }^{7}$. However, critical defects on EUVL mask blanks can occur with heights below $1 \mathrm{~nm}$ and thus a significant improvement in sensitivity will be required. A tool operating at $266 \mathrm{~nm}$ is under development and the expected increase in sensitivity at the shorter wavelength is calculated.

\footnotetext{
*EMGullikson@lbl.gov

SPIE 5751-146
} 
The roughness of the mask produces fluctuations in image contrast. These fluctuations combined with random noise limit the sensitivity of an inspection tool. It will be important to properly specify the roughness of production EUVL mask blanks to ensure that it does not compromise the ability to inspect for defects. With this in mind, the contrast fluctuations from a rough mask have been modeled and compared with measurements. The calculations are in good agreement with the observed rms contrast variations. The model may be used to help define the required roughness of future mask substrates.

\section{THEORY}

\subsection{Confocal imaging}

In a confocal microscope the mask is illuminated with the focused spatially coherent light from a point source. The reflected light is re-imaged onto a point detector. An image is formed by scanning the spot over the mask. The theory of the imaging of a scanning confocal microscope is described in papers by Sheppard ${ }^{8}$ and Wilson ${ }^{9}$. The image amplitude can be obtained from an integral over the scattering function $S(\hat{m}, \hat{n})$ where $\mathbf{m}$ and $\mathbf{n}$ are the unit vectors in the direction of the incident light and the scattered light respectively. ${ }^{10}$ The image field is given by

$$
U(\vec{x})=\int P_{1}(\hat{m}) P_{2}(\hat{n}) S(\hat{m}, \hat{n}) \exp (-i k(\hat{n}-\hat{m}) \cdot \vec{x}) d m_{x} d m_{y} d n_{x} d n_{y}
$$

where $P_{1}(\hat{m})$ is the pupil function for the incident radiation and $P_{2}(\hat{n})$ is the pupil function of the scattered radiation. Thus the image is based on the scattering function $S(\hat{m}, \hat{n})$ and the calculation of the scattering function for the various cases of interest is discussed in the following sections.
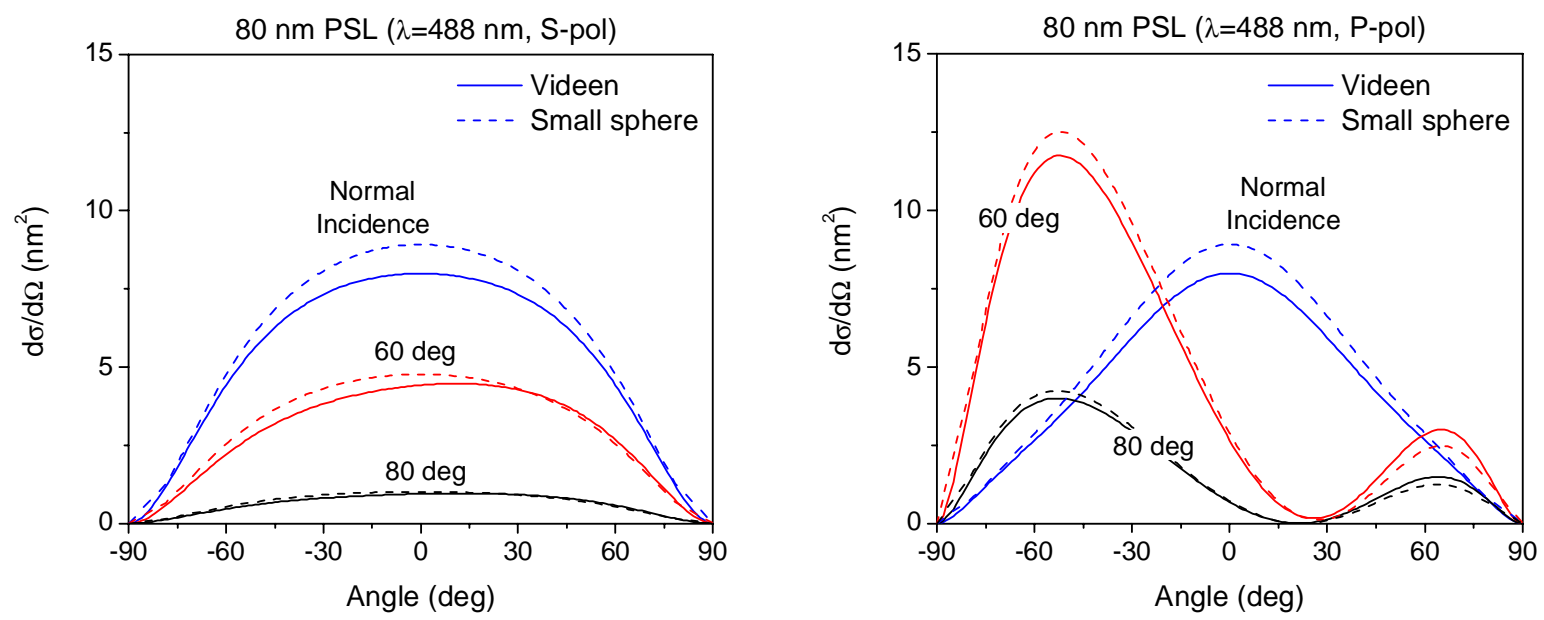

Figure 1. The differential scattering cross section for an $80 \mathrm{~nm}$ PSL sphere on fused silica for S-polarized and Ppolarized light. The three sets of curves are for light which is at normal incidence and 60 and 80 degrees from the normal. The solid lines are calculated with the method described by Videen and the dashed curves are using the dipole approximation.

\subsection{Scattering from a particle on a surface}


An approximate analytical solution for light scattering from a sphere above a surface was given by Videen ${ }^{11}$. The scattering solution is based on the Mie scattering of an isolated sphere. If the particle is small enough to be treated as a radiating dipole then calculation of the scattering amplitude can be greatly simplified ${ }^{12}$. In figure 1, the differential scattering cross section is shown for an $80 \mathrm{~nm}$ PSL sphere on fused silica for both S and P-polarized $488 \mathrm{~nm}$ light. The dipole approximation performs quite well for this case and it was used for the image calculations presented in this work. The confocal images were calculated with circularly polarized light.

Since the multilayer period of $7 \mathrm{~nm}$ is short compared with optical wavelengths, the Mo/Si multilayer on the mask blank was treated as a uniform medium with an effective index of refraction. At $488 \mathrm{~nm}$ an effective index of $\mathrm{n}=3.56, \mathrm{k}=2.59$ was used (as determined from reflectivity measurements). The attenuation length of $488 \mathrm{~nm}$ light at normal incidence would be $15 \mathrm{~nm}$ or just over 2 multilayer periods. At $266 \mathrm{~nm}$ an effective index of refraction of $\mathrm{n}=2.0$ and $\mathrm{k}=3.3$ was obtained from a weighted average of the optical constants of Mo and Si. The attenuation depth for $266 \mathrm{~nm}$ light in a $\mathrm{Mo} / \mathrm{Si}$ multilayer is $6.5 \mathrm{~nm}$ or just under one multilayer period.

\subsection{Scattering from a phase defect}

A rigorous solution of the scattering of visible light from a defect within the multilayer coating of a mask blank is possible but can be numerically intensive. A simple scalar single surface approximation (SSA) is adopted here. In this approximation the bump at the top surface of the multilayer is converted to a phase bump in the reflected wave with no change in the reflected wave amplitude. The perturbed wave is used as the input to the imaging calculation. This simple method is computationally fast and is sufficiently accurate for many cases of interest. The validity of the SSA has been investigated for EUV defect printability simulations ${ }^{13}$.

\section{MEASUREMENTS}

The measurements were performed on the Lasertec M1350 mask blank inspection tool installed at Intel in Santa Clara. This tool ${ }^{7}$ uses $488 \mathrm{~nm}$ light and was demonstrated to detect below $60 \mathrm{~nm}$ PSL spheres on fused silica substrate and 80 $\mathrm{nm}$ PSL spheres on $\mathrm{Mo} / \mathrm{Si}$ with a $>90 \%$ capture rate. The tool has a variable optical contrast enhancement capability which may be turned off to acquire images in a standard confocal mode.
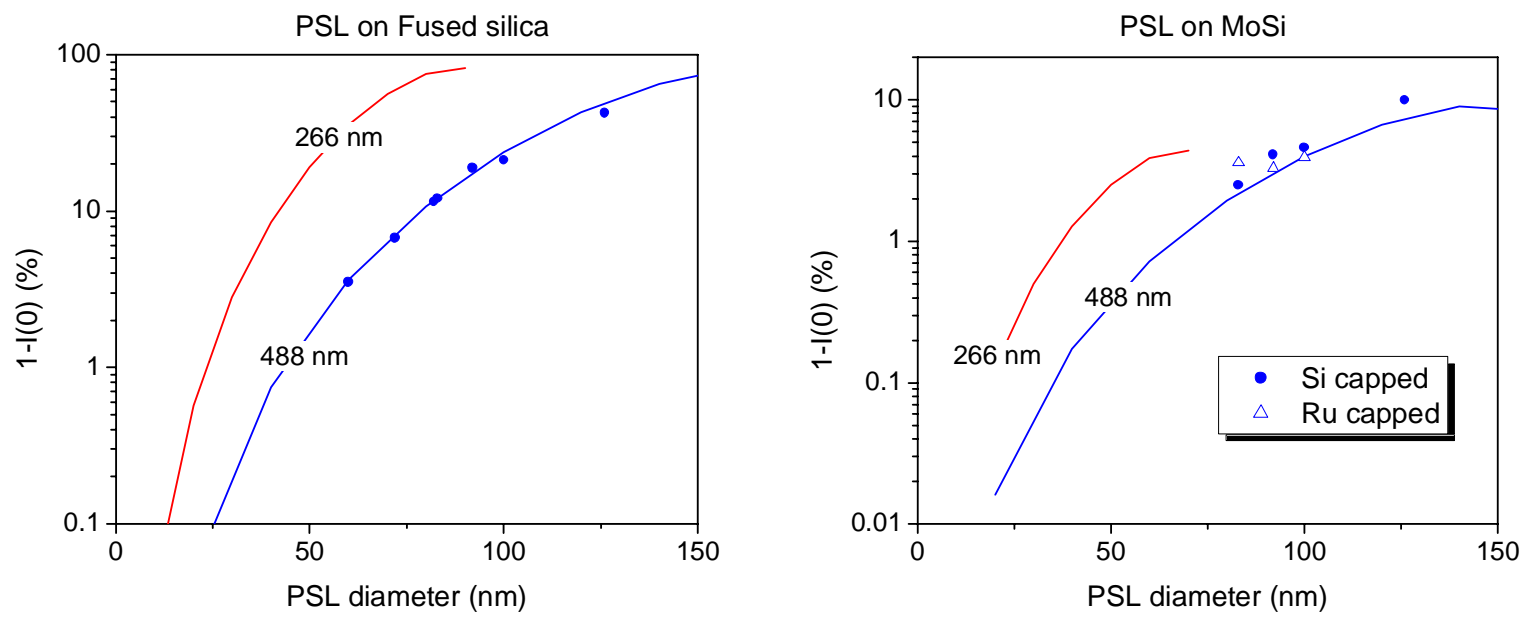

Figure 2. The image contrast in standard confocal mode of PSL spheres on a fused silica substrate and on a multilayer coated mask blank. The measurements are in excellent agreement with the calculated image contrast at $488 \mathrm{~nm}$. The predicted contrast for an identical microscope with $266 \mathrm{~nm}$ light is shown.

\subsection{Spherical particles}


PSL spheres were deposited on mask substrates and multilayer coated mask blanks. The measurements were performed with the M1350 in standard confocal mode, i.e. without optical contrast enhancement. The minimum in the review image is plotted versus PSL sphere diameter in Fig. 2. Measurements were performed on eight different sizes of PSL spheres on fused silica. Each point is the average contrast obtained from measurements of 30-40 spheres of each size. The measurements are all in excellent agreement with the predictions of the model with no adjustable parameters.

In figure 2, the contrast of PSL spheres on a multilayer coated mask blank is also shown. Due to the higher reflectivity from the multilayer, the image contrast is lower for a sphere on a multilayer coated substrate compared with a bare fused silica substrate. Measurements were performed with PSL spheres deposited on 2 mask blanks, the first with a multilayer with an $11 \mathrm{~nm} \mathrm{Si} \mathrm{capping} \mathrm{layer} \mathrm{and} \mathrm{the} \mathrm{other} \mathrm{with} \mathrm{a} 2.5 \mathrm{~nm}$ Ru capping layer. Measurements on both types of blanks show good agreement with the predictions of the model.

The calculated contrast at a wavelength of $266 \mathrm{~nm}$ is also shown in figure 2. The PSL diameter with a given contrast is found to scale proportional to the wavelength. For example, the PSL sphere diameter which gives a $2 \%$ contrast on fused silica decreases from $53 \mathrm{~nm}$ to $28 \mathrm{~nm}$. For the multilayer coated mask blank the PSL diameter at $2 \%$ contrast decreases from $81 \mathrm{~nm}$ to $47 \mathrm{~nm}$.

\subsection{Multilayer Defects}

Measurements were performed on programmed multilayer defects and reported in a previous paper ${ }^{7}$. In this section, the measured image contrast for the programmed defect mask V1522 is compared with the calculated image contrast using the single surface approximation described above. The programmed defects were fabricated using an electron beam nanowriter to create defects in HSQ resist on the mask substrate. The multilayer reflective coating was deposited on top of the programmed substrate defects. The height of the resulting bumps at the multilayer top surface ranged from $3 \mathrm{~nm}$ to $30 \mathrm{~nm}$ depending on the size of the substrate defect. The FWHM of the top surface bumps was in the range of 50-75 $\mathrm{nm}$. The measured contrast is shown in figure 3 for both the standard confocal and contrast enhanced images. The calculated contrast assuming a Gaussian defect shape with a FWHM of $60 \mathrm{~nm}$ is also shown. The agreement is good considering the significant uncertainty in the measurement of the FWHM from the AFM measurements due to tip size effects.

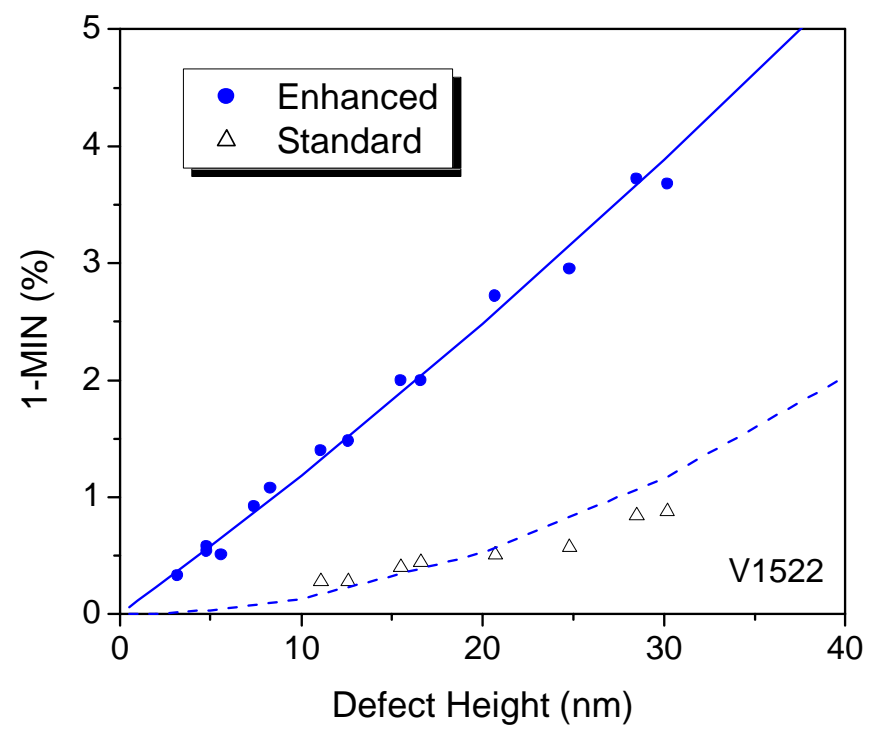

Figure 3. The image contrast of multilayer programmed defects measured in both the standard confocal and the contrast enhanced modes. The model calculations assume a Gaussian defect profile with a FWHM $=60 \mathrm{~nm}$. 
It was shown in reference 7 that multilayer defects as small as 10-12 $\mathrm{nm}$ high could be detected in the contrast enhanced mode with a capture rate greater than $90 \%$. The contrast of $10-12 \mathrm{~nm}$ high defects with FWHM=60 nm is in the range of 1.2-1.4\%. The minimum detectable contrast is determined by the system noise and the contrast fluctuations due to the roughness of the mask blank. The effect of mask roughness is discussed in the next section.

\subsection{Roughness}

The RMS contrast variation due to roughness was measured for three mask blanks on substrates with varying roughness. The results for the three masks are summarized in Table 1. The high spatial frequency roughness (HSFR) was determined from AFM measurements by integrating the Power Spectral Density (PSD) over spatial periods from 50 $\mathrm{nm}$ to $250 \mathrm{~nm}$. The slope error was determined from AFM measurements after low pass filtering with a cutoff frequency of $1 /(250 \mathrm{~nm})$. The HSRF describes the roughness over length scales which scatter light out of the entrance aperture of the EUV imaging optics and leads to a loss in throughput. Roughness in the frequency range described by the slope error can lead to line edge roughness (LER) in the printed image. A proposed specification for high frequency slope errors is $1.8 \mathrm{mrad}(3 \sigma)$. Only the smooth FS substrate has a slope error close to meeting this requirement.
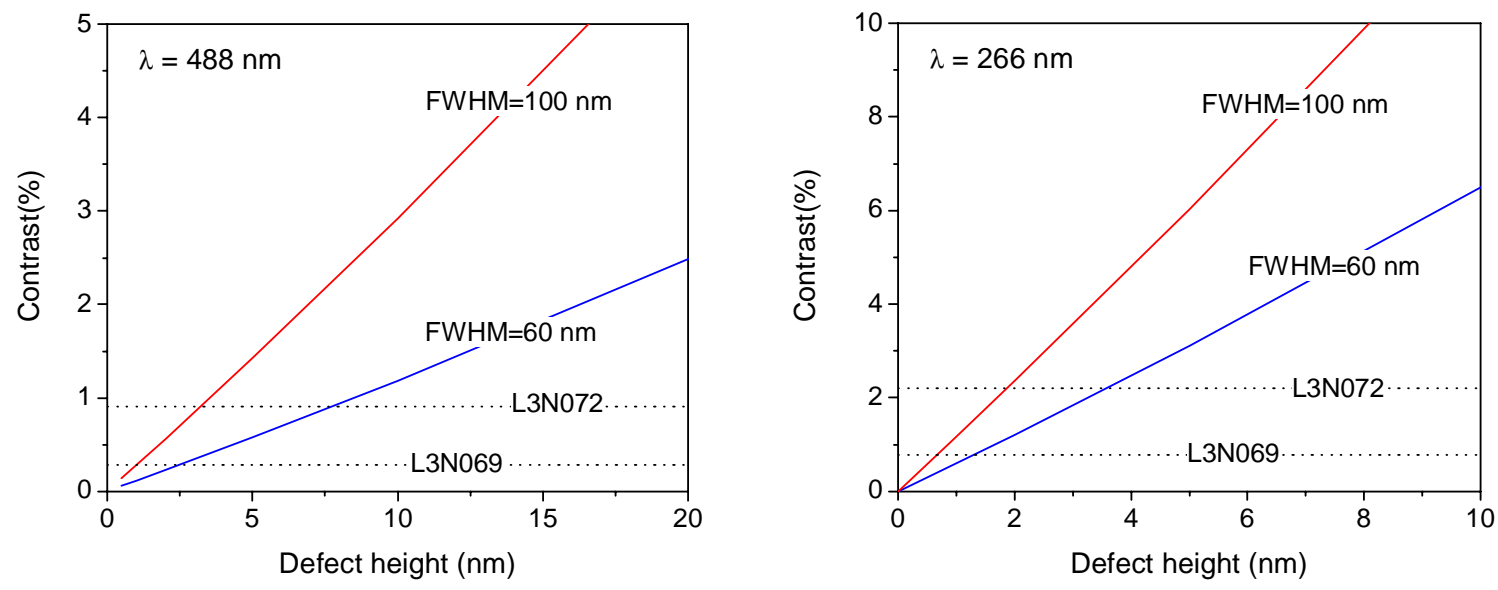

Figure 4. The calculated image contrast of multilayer defects versus peak height for the M1350 at $488 \mathrm{~nm}$ and a similar microscope operating at $266 \mathrm{~nm}$ with contrast enhancement. The dashed lines are at $7 \times$ the RMS contrast fluctuations due to roughness for two mask blanks.

Ten review images were obtained at each of five locations on the three mask blanks. The images were taken in the standard confocal mode and in the contrast enhanced mode. The random noise was determined from the difference in the repeated images and was about 0.29\% RMS for a single measurement. By taking the difference between measurements performed at two different locations on a mask blank any repeatable instrumental effects are canceled out and only the real contrast variations and random noise remain. By averaging over the 10 repeated measurements it was possible to measure the contrast variations of below $0.1 \%$ RMS. The RMS contrast, measured in this way, is shown in Table 1.

\begin{tabular}{|l|l|l|l|l|}
\hline Mask & HSFR & $3 \sigma$ slope & $\begin{array}{l}\text { Contrast } \\
\text { Measured }\end{array}$ & $\begin{array}{l}\text { Contrast } \\
\text { Modeled }\end{array}$ \\
\hline L3N072 & $0.19 \mathrm{~nm}$ & $5.5 \mathrm{mrad}$ & $0.12 \%$ & $0.12 \%$ \\
\hline L3N066 & $0.15 \mathrm{~nm}$ & $4.3 \mathrm{mrad}$ & $0.10 \%$ & $0.10 \%$ \\
\hline L3N069 & $0.11 \mathrm{~nm}$ & $2.0 \mathrm{mrad}$ & $\sim 0.03 \%$ & $0.04 \%$ \\
\hline
\end{tabular}

Table 1. Results for the three mask blanks used to study the imaging of roughness. 
In order to model the contrast fluctuations a surface was numerically generated using the PSD determined from AFM measurements. Image contrast was calculated using the simple single surface approximation described above. In the standard confocal mode, the intensity fluctuations due to roughness were too small to be measured, which is consistent with the simulation results. Excellent agreement is obtained between the calculated and measured RMS contrast variation for each of the mask blanks in the contrast enhanced mode, see Table 1.

The model was used to calculate the image contrast for the same microscope operating at a wavelength of $266 \mathrm{~nm}$. It was found that the contrast variation resulting from the roughness increased for all three masks by between 2.5-3 times. In figure 4, the calculated contrast for Gaussian multilayer defects is shown. The dashed lines are at 7-sigma where sigma is the calculated RMS roughness induced intensity fluctuations and are meant to indicate the limit to the sensitivity due to mask roughness.

\section{CONCLUSIONS}

A model has been developed for the image contrast of a confocal microscope. The predictions of the model for particles on a surface have been validated with measurements at a wavelength of $488 \mathrm{~nm}$ using PSL spheres on fused silica substrates and on multilayer coated mask blanks. Excellent agreement is obtained with no adjustable parameters used in the model. The model also shows good agreement with measurements for programmed multilayer defects. The contrast fluctuations produced by the roughness were also calculated for three mask blanks with varying surface roughness. With the microscope operating in contrast enhanced mode the roughness induced contrast fluctuations were observable and in excellent agreement with the model predictions based on AFM measurements.

The expected response of a microscope operating with $266 \mathrm{~nm}$ light was calculated. For PSL spheres it was found that for a given image contrast the diameter scales with the wavelength. So for example, the PSL sphere diameter which gives a $2 \%$ contrast can be expected to decrease from $53 \mathrm{~nm}$ to $28 \mathrm{~nm}$ on fused silica and from $81 \mathrm{~nm}$ to $47 \mathrm{~nm}$ on a multilayer mask blank. The contrast fluctuations due to mask roughness increased at $266 \mathrm{~nm}$ by 2.5-3 times for the masks considered. The roughness will need to be reduced on the substrates for EUVL masks and future modeling work will help to formulate a specification based on the requirements of visible light defect inspection.

\section{ACKNOWLEDGEMENTS}

The authors would like to thank Anton Barty and Ted Liang for many helpful discussions. The programmed defect mask was fabricated by Alex Liddle, LBNL and Paul Mirkarimi, LLNL. The PSL samples were prepared by the Scatterworks. This work was funded by Intel Corporation.

\section{REFERENCES}

\footnotetext{
${ }^{1}$ Yoshihiro Tezuka, Masaaki Ito, Tsunea Terasawa, Toshihisa Tomie, "Actinic Detection and Signal Characterization of Multilayer Defects on EUV Mask Blanks.” Proc. SPIE 5567, 791-799 (2004).

${ }^{2}$ A. Barty, Y. Liu, E.M. Gullikson, “Actinic inspection of multilayer defects on EUV masks," SPIE 5751-78 (2005).

${ }^{3}$ A.R. Stivers, T. Liang, M. Penn, B. Lieberman, G. Sheldon, J. Folta, C. Larson, P. Mirkarimi, C. Walton, E.M. Gullikson, M. Yi, "Evaluation of the capability of a multi-beam confocal inspection system for inspection of EUVL mask blanks", Proc. SPIE 4889, 408-417 (2002).

${ }^{4}$ Alan R. Stivers, Pei-Yang Yan, Guojing Zhang, Ted Liang, Emily Y. Shu, Edita Tejnil, Barry Lieberman, Rajesh Nagpal, Kangmin Hsia, Michael Penn and Fu-Chang Lo, "EUV Mask Pilot Line at Intel Corporation." Proc. SPIE 5567, 13-22 (2004).

${ }^{5}$ J.-P. Urbach, J. Cavelaars, H. Kusunose, T. Liang, A.R. Stivers, "EUV substrate and blank inspection with confocal microscopy" Proc. SPIE 5256, 556-65 (2003).

${ }^{6}$ E.M. Gullikson, Edita Tejnil, Ted Liang, Alan R. Stivers, "EUVL defect printability at the 32-nm node", Proc. SPIE 5374, $791-796$ (May 2004).
} 
${ }^{7}$ Edita Tejnil, Eric M. Gullikson, Alan R. Stivers, "Characterization of defect detection sensitivity in inspection of mask substrates and blanks for extreme-ultraviolet lithography." Proc. SPIE Vol. 5567, 943-952 (Dec. 2004).

${ }^{8}$ C.J.R. Sheppard and A. Choudhury, "Image formation in the scanning microscope," Optica Acta 24, 1051-1073 (1977).

${ }^{9}$ T. Wilson, "Confocal Microscopy," in Confocal Microscopy edited by T. Wilson (Academic Press 1990).

${ }^{10}$ C.J.R. Sheppard, T.J. Connolly, Min Gu, "Imaging and reconstruction for rough surface scattering in the Kirchhoff approximation by confocal microscopy," J. Mod. Optics 40, 2407-2421 (1993).

${ }^{11}$ G. Videen, "Light scattering from a sphere on or near a surface," J. Opt. Soc. Am. A 8, 483 (1991).

${ }^{12}$ G. Videen, M.G. Turner, V.J. Iafelice, W.S. Bickel, W.L. Wolfe, "Scattering from a small sphere near a surface," J. Opt. Soc. Am. A 10, 118 (1993).

${ }^{13}$ E.M. Gullikson, C. Cerjan, D.G. Stearns, P.B. Mirkarimi, D.W. Sweeney, "A practical approach for modeling EUVL mask defects." J. Vac. Sci. Technol. B 20, 81 (2002). 